\title{
los álcalis en la estructura del clínker de cemento portland
}

\begin{abstract}
Se pone de relieve la influencia de los álcalis en la constitución del clinker, asi como en la composición potencial ael mismo, según su interacción con otros componentes mayores y menores, y de acuerdo con los sistemas de equilibrio que se establecen en el proceso de fabricación, dependientes de la composición del crudo $\mathrm{y}$ de las condiciones de cocción y enfriamiento.
\end{abstract}

Se estudia experimentalmente la distribución de álcalis en un clínker granulado cocido con fuel-oll en un horno Lepol, y se comparan los resultados con los de otras investigaciones sobre la estructura general del material anteriormente efectuadas. Se discute la influencia de dicha distribución en la constitución de los gránulos de clínker.

Se incluye una amplla relación bibliográftca de los trabajos más destacados y recientes acerca de la reacción expansiva árido-álcalis en los hormigones.

\section{INTRODUCCION (1) (2) (3).}

Entre los comúnmente llamados componentes menores del clínker de cemento portland se encuentran los alcalis, es decir, los óxidos sódico $y$ potásico. Su presencia en el clínker se debe a su existencia en las materias primas del crudo, particularmente en las de tipo arcilloso. Otra aportación de álcalis al clínker es la producida por las cenizas del combustible utilizado en la cocción del crudo, cuando se trata de carbón.

No todos los álcalis existentes en el crudo y los aportados a éste por las cenizas del combustible se conservan despues en el clínker, pues una buena parte de ellos se volatiliza en la zona de clinkerización del horno. De esta parte, otra, a su vez, se elimina con los gases de la chimenea, mientras que el resto se reincorpora al crudo en las partes más frías del horno o queda en el polvo separado de los gases de combustión, el cual frecuentemente se vuelve a introducir en el horno mezclado con nuevo crudo (4) (5).

La cantidad de cenizas de carbón que quedan incorporadas al clínker, si bien se puede cifrar por término medio en $2 / 3$ de su totalidad, depende de hecho del tipo de horno, por lo cual también depende del mismo la proporción de álcalis aportados al clínker por dichas cenizas.

La volatilización y eliminación de álcalis, ya procedan de cenizas o ya se encuentren en las materias primas que constituyen el crudo, varía igualmente con la clase de horno $\mathrm{y}$ con el tipo de proceso, así como con la naturaleza de los materiales.

La eliminación es distinta para el óxido potásico que para el sódico, por cuanto que el primero es mucho más volátil que el segundo, y por ello se vaporiza a menor temperatura y en mayor proporción.

De acuerdo con estos hechos se suele considerar que la eliminación alcanza de $15-60 \%$ de $\mathrm{Na}_{2} \mathrm{O}$ y al $40-80 \%$ de $\mathrm{K}_{2} \mathrm{O}$ en 
vía seca, y al $10-30 \%$ de $\mathrm{Na}_{2} \mathrm{O}$ y al $20-50 \%$ de $K_{2} O$ en vía húmeda; en términos más generales, se admiten las cifras de $20 \%$ para el $\mathrm{Na}_{2} \mathrm{O}$ y $50 \%$ para el $\mathrm{K}_{2} \mathrm{O}$.

Los hornos más largos, lógicamente retienen mayor proporción de álcalis; tal es el caso de la vía húmeda en comparación con la via seca.

Cuanto mayor es la temperatura de clinkerización a mayor es el peso del litro de clínker obtenido (ambos factores están directamente relacionados), menor es la cantidad de álcalis que éste contiene.

La eliminación de los mismos es más difícil y, por lo tanto, menor, en los casos en que el crudo contiene sulfatos; entonces es también mayor cantidad de álcalis solubles del clínker (6). La introducción - la presencia de sulfato cálcico en los crudos Ilega a inhibir la volatilización del óxido potásico.

Los álcalis, si bien son componentes menores del clínker, tienen su importancia, tanto en el proceso de fabricación de éste como en su estructura (7) (8), por lo cual se ha prestado la debida atención al comportamiento de los mismos en el horno (9). En cuanto al proceso, la formación de gránulos a partír del material crudo pulverulento tiene lugar a una cierta temperatura que depende de la cantidad de fase líquida correspondiente a la misma, y de la viscosidad y tensión superficial de dicha fase líquida. En una y otras influye la presencia de óxido sódico, rebajando la temperatura, aumentando la proporción del material fundido y disminuyendo la viscosidad de éste. El óxido potásico, por su mayor volatilidad, ejerce una influencia mucho menor como fundente. Por las mismas razones los álcalis $\mathrm{y}$, en particular, el óxido sódico, actúan como causa de la formación de anillos de crudo en los hornos y del ataque al material refractario de los mismos.

Por lo que se refiere a la estructura del clínker, los álcalis son capaces de formar combinaciones con otros componentes, las cuales, a su vez, dan lugar, con algunos de los constituyentes, a otras especies distintas y a disoluciones sólidas, todo lo cual se traduce en una modificación de la com posición potencial del clínker, en relación con la que resultaría de un cálculo que no tuviese en cuenta los álcalís.

Estos se encuentran en el clínker en proporciones distintas, siendo mayor en general la cantidad de óxido potásico que la de óxido sódico. Se hallan preferentemente combinados con el anhidrido sulfúrico, formando una disolución sólida de sulfato sódico-potásico, cuya composición tiende a ser la correspondiente a la fórmula:

$$
3 \mathrm{~K}_{2} \mathrm{SO}_{4} \cdot \mathrm{Na}_{2} \mathrm{SO}_{4} \text { o blen } \mathrm{K}_{0,75} \mathrm{Na}_{6,25} \mathrm{SO}_{4} .
$$

Generalmente sucede así, independientemente de la relación $\mathrm{K}_{2} \mathrm{O} / \mathrm{Na}_{2} \mathrm{O}$, cuando la suma de óxidos sódico y potásico, expresada en moles, es del orden del doble al triple de la cifra correspondiente al $\mathrm{SO}_{3}$.

El contenido de éste en el clínker es pequeño (suele oscilar de 0,1 a $0,5 \%$ ) y en general insuficiente para fijar la totalidad de álcalis, en forma del sulfato doble con la composición señalada.

El exceso de álcalis puede permanecer en la fase vítrea, si el enfriamiento del clínker es lo bastante rápido. En el caso extremo contrario, es decir, cuando el enfriamiento es suficientemente lento para permitir una cristalización completa, los álcalis forman los compuestos $\mathbf{K C}_{23} \mathbf{S}_{12}$ y $\mathrm{NC}_{\mathrm{s}} \mathbf{A}_{3}$; el primero de ellos no es miscible con la fase líquida. El segundo puede formar disolución sólida con el $\mathbf{C}_{8} A$ el $C_{12} A_{7}$ (intersticial prismático oscuro) $e$ incluso con el $\mathrm{C}_{2} \mathbf{S}$.

También se encuentra en el clínker que contiene álcalis el $\mathbf{N C}_{8} \mathbf{A}_{3}$, análogo aI $\mathrm{KC}_{\mathrm{s}} \mathbf{A}_{3}$, pero menos estable que éste, dado el mayor radio iónico deI potásico. El $\mathrm{NC}_{5} A_{3}$ puede existir en el sistema $\mathrm{C}_{8} \mathrm{~S}-\mathrm{C}_{2} \mathrm{~S}-\mathrm{C}_{7} \mathrm{~A}-\mathrm{C}_{4} \mathrm{AF}$ y fase líquida, para contenidos de hasta $1 \%$ de $\mathrm{Na}_{2} \mathrm{O}$. Como el $\mathbf{N C}_{8} \mathbf{A}_{8}$, el $\mathbf{K C}_{8} \mathbf{A}_{3}$ parece formar parte del intersticial prismático oscuro.

Lo anteriormente expuesto Indica que el óxido potásico sustituye a un mol de $\mathrm{CaO}$ en cada 12 moles de $\mathrm{C}_{2} \mathrm{~s}, \mathrm{y}$ que el 
óxido sódico sustituye a otro mol de $\mathrm{CaO}$ en cada tres moles de $C_{3} A$.

Por lo tanto, los álcalis del clínker se pueden encontrar en dos formas: como álcalis solubles en forma de sulfatos o de sulfato doble (disolución sóltda) con la composición indicada, en la que el óxido potásico participa en mayor proporción que el sódico, y formando combinaciones con los silicatos y aluminatos (el óxido potásico preferentemente con el silicato bicálcico y el oxido sódico con el aluminato tricálcico).

Con más precisión puede indicarse que en el sistema constituído por el clínker de cemento portland, además de los constituyentes principales $\mathrm{C}_{8} \mathrm{~S}-\mathrm{C}_{2} \mathrm{~S}-\mathrm{C}_{8} \mathrm{~A}-$ $-C_{a} A F$, pueden darse los siguientes:

a) Si hay $\mathrm{SO}_{3}$ en exceso sobre los álcalis:

$$
\mathrm{K}_{2} \mathrm{SO}_{4} ; \mathrm{Na}_{2} \mathrm{SO}_{4} \text { y } \mathrm{CaSO}_{4} \text {. }
$$

b) Si hay álcalis en exceso sobre el $\mathrm{SO}_{3}:$

$$
3 \mathrm{~K}_{2} \mathrm{SO}_{4} \cdot \mathrm{Na}_{2} \mathrm{SO}_{4} \text {. }
$$

y

$b_{1}$ ) Si hay exceso de $K_{2} O$ :

$$
\mathrm{K}_{2} \mathrm{SO}_{4} \text { y } \mathrm{KC}_{29} \mathbf{S}_{12} \text {. }
$$

$b_{2}$ ) Si hay exceso de $\mathrm{Na}_{2} \mathrm{O}$ :

$$
\mathrm{Na}_{2} \mathrm{SO}_{4} \text { y } \mathrm{NC}_{8} \mathrm{~A}_{3} .
$$

$b_{3}$ ) Si hay exceso de $\mathrm{Na}_{2} \mathrm{O}$ y $\mathrm{K}_{2} \mathrm{O}$ :

$$
\mathrm{KC}_{23} \mathrm{~s}_{12} \text { y } \quad \mathrm{NC}_{8} \mathrm{~A}_{3} \text {. }
$$

A partir de estos datos existen fórmulas para efectuar el cálculo de la composición potencial del clínker, teniendo en cuenta las correcciones que, debido a los álcalis, es preciso introducir en las fórmulas de Bogue. En este sentido, parece ser que tanto el $\mathrm{K}, \mathrm{O}$ como el $\mathrm{Na}_{2} \mathrm{O}$ elevan el contenido de $\mathrm{C}_{3} \mathrm{~S}$, rebajan el de $\mathrm{C}_{2} \mathrm{~S}$ y no modifican el de $C_{4} A F$; el $K_{2} O$ no modifica el contenido de $\mathrm{C}_{3} \mathrm{~A}$ y el $\mathrm{Na}_{22} \mathrm{O}$ lo rebaja.

En cuanto al mecanismo de estas acciones puede aceptarse la siguiente explicación.
En el sistema $\mathrm{CaO}$ (C)- $\mathrm{Al}_{2} \mathrm{O}_{3}$ (A) $-\mathrm{SiO}_{2}$ (S) $-\mathrm{Fe}_{2} \mathrm{O}_{3}(\mathrm{~F})-\mathrm{K}_{2} \mathrm{O}(\mathrm{K})$, la formación del $\mathrm{KC}_{28} \mathbf{S}_{1 \mathrm{i}}$ puede considerarse que transcurre según la reacción

$$
12 \mathrm{C}_{2} \mathrm{~S}+\mathrm{K}_{2} \mathrm{O} \rightarrow \mathrm{KC}_{23} \mathrm{~S}+\mathrm{CaO},
$$

en la cual se produce cal libre (procedente del $\mathrm{C}_{2} \mathrm{~s}$ ), que puede combinarse con más silicato bicálcico para formar sillicato tricálcico. En este sentido, el óxido potásico puede contribuir a elevar el contenido de $\mathrm{C}_{3} \mathrm{~S}$ y a rebajar el de $\mathrm{C}_{2} \mathrm{~S}$.

Por otra parte, el óxido sódico puede reaccionar con el $\mathrm{C}_{3} \mathrm{~S}$, descomponiéndolo en $\mathrm{C}_{2} \mathrm{~S}$ y $\mathrm{CaO}$ (procedente del $\mathrm{C}_{3} \mathrm{~S}$ ), lo que contribuye a aumentar la cal libre en clínkeres que proceden de crudos ricos en $\mathrm{Na}_{2} \mathrm{O}$. De hecho, al enfriar en condiciones de equilibrio ciertas composiciones en presencia de óxido sódico, contienen cal libre que no tendrían en ausencia del mismo, ya que éste ensancha el campo del $\mathrm{CaO}$ y, en tal sentido, puede contribuir a elevar la cal libre del clínker y el $\mathrm{C}_{p} \mathrm{~S}$, disminuyendo el $\mathrm{C}_{3} \mathrm{~S}$.

Pero, en otro aspecto, lo más probable es que, como queda indicado, se produzca la reacción

$$
3 \mathrm{C}_{3} \mathrm{~A}+\mathrm{Na}_{2} \mathrm{O} \rightarrow \mathrm{NC}_{8} \mathrm{~A}_{3}+\mathrm{CaO} .
$$

en la que igualmente se produce cal libre que, combinada con el silicato bicálcico, eleva la cifra de silicato tricálcico a expensas de disminuir el contenido de aquél.

$\mathrm{Si}$ bien la especie $\mathbf{K C}_{2 \mathrm{~g}} \mathrm{~S}_{12}$ es muy similar al $\mathbf{C}_{2} \mathbf{S}$, presenta con relación a ella ciertas características diferenciales. Así, por ejemplo, un contenido de $0,5 \%$ de $\mathrm{KC}_{23} \mathrm{~S}_{12}$ acelera la hidratación de los constituyentes del cemento y particularmente la del propio $\mathrm{C}_{2} \mathrm{~S}$. Por otro lado, así como el silicato bicálcico es susceptible de combinarse con cal libre para formar silicato tricálcico, el $\mathbf{K C}_{23} \mathbf{S}_{12}$ no lo es. A esto último se debe el que, en realidad, la presencia de óxido potásico haga disminuir el contenido máximo posible de $\mathrm{C}_{3} \mathrm{~S}$. En efecto, $1 \%$ de $\mathrm{K}_{2} \mathrm{O}$ reacciona con $21,9 \%$ de $\mathbf{C}_{2} \mathbf{S}$, para dar $22,3 \%$ de $\mathbf{K C}_{28} \mathbf{S}_{12}$ y $0,6 \%$ de CaO libre que puede combinarse con $1,9 \%$ de $\mathbf{C}_{2} \mathrm{~S}$ y formar $2,5 \%$ dè $\mathrm{C}_{8} \mathrm{~S}$. 
De aquí que cada $1 \%$ de $K_{2} O$ elimine $23,8 \%$ de $\mathrm{C}_{2} \mathrm{~S}$, y si el contenido de dicho constituyente en el clínker es igual o menor que $23,8 \%$, queda sin transformar en $\mathrm{C}_{3} \mathrm{~S} \mathrm{y}$, por consiguiente, el $0,6 \%$ de cal libre no se combina y permanece como tal.

Es más, suponiendo que no existiera suficiente cantidad de silicato bicálcico para reaccionar con el $\mathrm{K}_{2} \mathrm{O}$, éste descompondría al silicato tricálcico según la reacción

$$
\begin{gathered}
12\left(3 \mathrm{CaO} \cdot \mathrm{SiO}_{2}\right)+\mathrm{K}_{2} \mathrm{O} \\
\rightarrow \mathrm{K}_{2} \mathrm{O} \cdot 23 \mathrm{CaO} \cdot 12 \mathrm{SiO}_{2}+13 \mathrm{CaO},
\end{gathered}
$$

de la cual se deduce que $1 \%$ de $\mathrm{K}$, O rebaja en un $29,1 \%$ el contenido máximo posible de $\mathrm{C}_{3} \mathrm{~S}$ y libera $7,7 \%$ de $\mathrm{CaO}$. La lentitud de la reacción

$$
\mathrm{C}_{2} \mathrm{~S}+\mathrm{CaO} \rightarrow \mathrm{C}_{3} \mathrm{~S}
$$

contribuye también a que la cantidad de cal libre observada sea siempre mayor que la cantidad correspondiente a las condiciones de equilibrio.

La influencia más considerable en la constitución del clínker es, pues, la ejercida por el óxido potásico, y se manifiesta más ostensiblemente en clínkeres de contenido elevado de cal (abundantes en $\mathbf{C}_{3} \mathbf{S}$ ). La magnitud de dicha influencia depende

$r \div 1,0$

$$
\begin{aligned}
& \mathrm{O}<\mathrm{K}_{c}<\frac{0,75}{T} \\
& \frac{0,75}{r} \\
& \mathbf{K}_{5}=\mathbf{K}_{\text {。 }} \\
& \mathbf{K}_{s}=r . \mathbf{K}_{c} \\
& \mathbf{K}_{s}=\mathbf{0 , 7 5}
\end{aligned}
$$

de la cantidad de $K_{2} O$ disponible para la formación de $\mathrm{KC}_{2 \mathrm{~g}} \mathrm{~S}_{12}$, que depende, a su vez, de la proporción de álcalis remanente en disolución sólida con otros compuestos, Ia cual es función de las condiciones más o menos próximas a las de equilibrio durante el enfriamiento del clínker.

En el caso extremo ideal de una cristalización completa en condiciones de equilibrio (enfriamiento lentísimo del clínker) existen ecuaciones para el cálculo de la composición potencial en cada uno de Ios cuatro sistemas posibles antes indicados, en los que participan los álcalis (2). El sistema que se establece en cada caso depende de la relación molar $\mathrm{R}_{2} \mathrm{O}_{3}: \mathrm{SO}_{3}$ en el clínker:

$$
r=\frac{0,8500 \mathrm{KK}_{2} \mathrm{O}+1,2915 \mathrm{Na}_{2} \mathrm{O} \%}{\mathrm{SO}_{\mathrm{i}} \%}
$$

de la fracción molar del $K_{2} O$ en la totalidad de álcalis del clínker:

$$
\mathrm{K}_{e}=\frac{0,8500 \mathrm{~K}_{2} \mathrm{O} \%}{r \cdot \mathrm{SO}_{\mathrm{a}} \%}
$$

$\mathrm{y}$ de la fracción molar del $\mathrm{K}_{2} \mathrm{SO}_{4}$ en el sulfato doble, $\mathbf{K}_{s}$, cuyo valor se calcula así:

$$
r>1,0
$$

Para $r$ 1,0 puede darse el sistema

$$
\mathrm{C}_{3} \mathrm{~B}-\mathrm{C}_{2} \mathrm{~S}-\mathrm{C}_{3} \mathrm{~A}-\mathrm{C}_{4} \mathrm{AF} \text {, }
$$

con la siguiente composición potencial:

$$
\begin{aligned}
& \mathrm{C}_{9} \mathrm{~S}=4,07 \mathrm{CaO}-6,72 \mathrm{Al}_{2} \mathrm{O}_{3}-1,43 \mathrm{Fe}_{2} \mathrm{O}_{3}-7,60 \mathrm{SlO}_{2}- \\
& \quad 2,85 \mathrm{SO}_{5}+3,68 \mathrm{Na}_{2} \mathrm{O}+2,42 \mathrm{~K}_{2} \mathrm{O} \\
& \mathrm{C}_{2} \mathrm{~S}=5,07 \mathrm{Al}_{2} \mathrm{O}_{3}+1,08 \mathrm{Fe}_{2} \mathrm{O}_{3}+8,60 \mathrm{SiO}_{2}+2,15 \mathrm{SO}_{3}- \\
& -2,78 \mathrm{Na}_{2} \mathrm{O}-1,83 \mathrm{~K}_{2} \mathrm{O}-3,07 \mathrm{CaO}
\end{aligned}
$$

$$
\begin{aligned}
& \mathrm{C}_{3} \mathrm{~A}=2,65 \mathrm{Al}_{2} \mathrm{O}_{3}-1,69 \mathrm{Fe}_{2} \mathrm{O}_{3} \\
& \mathrm{C}_{4} \mathrm{AF}=3,04 \mathrm{Fe}_{2} \mathrm{O}_{3} \\
& \mathrm{CaSO}_{4}=1,70 \mathrm{SO}_{3}-2,20 \mathrm{Na}_{2} \mathrm{O}-1,45 \mathrm{~K}_{2} \mathrm{O} \\
& \mathrm{Na}_{2} \mathrm{SO}_{4}=2,29 \mathrm{Na}_{2} \mathrm{O} \\
& \mathrm{K}_{2} \mathrm{SO}_{4}=1,85 \mathrm{~K}_{2} \mathrm{O}
\end{aligned}
$$

o bien el sistema

$$
\mathrm{CaO}-\mathrm{C}_{3} \mathrm{~S}-\mathrm{C}_{3} \mathrm{~A} \quad \mathrm{C}_{4} \mathrm{AF},
$$


con la siguiente composición potenctal:

$$
\begin{aligned}
& \mathrm{CaO} \text { libre }=\mathrm{CaO}-1,65 \mathrm{Al}_{2} \mathrm{O}_{8}-0,35 \mathrm{Fe}_{2} \mathrm{O}_{3}- \\
& -2,80 \mathrm{SiO}_{2}-0,70 \mathrm{SO}_{3}+0,90 \mathrm{Na}_{2} \mathrm{O}+0,60 \mathrm{~K}_{2} \mathrm{O} \\
& \mathrm{C}_{8} \mathrm{~S}=3,80 \mathrm{SiO}_{2} \\
& \mathrm{C}_{3} \mathrm{~A}=2,65 \mathrm{Al}_{2} \mathrm{O}_{3}-1,69 \mathrm{Fe}_{2} \mathrm{O}_{3} \\
& \mathrm{C}_{4} \mathrm{AF}=3,04 \mathrm{Fe}_{2} \mathrm{O}_{3} \\
& \mathrm{CaSO}_{4}=1,70 \mathrm{SO}_{3}-2,20 \mathrm{Na}_{2} \mathrm{O}-1,45 \mathrm{KK}_{2} \mathrm{O} \\
& \mathrm{Na}_{2} \mathrm{SO}_{4}=2,29 \mathrm{Na}_{2} \mathrm{O} \\
& \mathrm{K}_{22} \mathrm{SO}_{4}=1,85 \mathrm{~K}_{2} \mathrm{O}
\end{aligned}
$$

Para $r>1,0$ puede darse el sistema

$$
\mathrm{C}_{\mathrm{s}} \mathrm{B}-\mathrm{C}_{2} \mathrm{~B}-\mathrm{C}_{\mathrm{g}} \mathrm{A}-\mathrm{C}_{4} \mathrm{AF} \text {, }
$$

con la siguiente composición potencial:

$$
\begin{aligned}
& \mathrm{C}_{3} \mathrm{~S}=4,07 \mathrm{CaO}-6,72 \mathrm{Al}_{2} \mathrm{O}_{3}-1,43 \mathrm{Fe}_{2} \mathrm{O}_{3}-7,60 \mathrm{SiO}_{2} \\
& -2,85 \mathrm{SO}_{\mathrm{r}}+3,68 \mathrm{Na}_{2} \mathrm{O}+2,42 \mathrm{~K}_{2} \mathrm{O} \\
& \mathrm{C}_{28} \mathrm{~S}=5,07 \mathrm{Al}_{2} \mathrm{O}_{3}+1,08 \mathrm{Fe}_{2} \mathrm{O}_{3}+8,60 \mathrm{SiO}_{2}+ \\
& +2,15\left(12 \mathrm{~K}_{s}+1\right) \mathrm{SO}_{3}-2,78 \mathrm{Na}_{2} \mathrm{O}-23,77 \mathrm{~K}_{2} \mathrm{O} \\
& -3,07 \mathrm{CaO} \\
& \underset{3-13,07 \mathrm{Na}_{2} \mathrm{O}}{\mathrm{C}_{3} \mathrm{~A}=2,65 \mathrm{O}_{5}-1,69 \mathrm{Fe}_{2} \mathrm{O}_{3}+10,12\left(1-\mathrm{K}_{s}\right) \mathrm{SO}_{3}-} \\
& \mathrm{C}_{4} \mathrm{AF}=3,04 \mathrm{Fe}_{2} \mathrm{O}_{\mathrm{B}} \\
& \mathrm{NC}_{8} \mathrm{~A}_{3}=13,17 \mathrm{Na}_{2} \mathrm{O}-10,20\left(1-\mathrm{K}_{5}\right) \mathrm{SO}_{3} \\
& \mathrm{KC}_{23} \mathrm{~S}_{1 \mathrm{z}}=22,35 \mathrm{~K}_{2} \mathrm{O}-26,30 \mathrm{~K}_{3} . \mathrm{SO}_{3} \\
& \mathrm{Na}_{2} \mathrm{SO}_{4}=1,77\left(1-\mathrm{K}_{s}\right) \mathrm{SO}_{9} \\
& \mathrm{~K}_{2} \mathrm{SO}_{4}=2,18 \mathrm{~K}, . \mathrm{SO}_{3}
\end{aligned}
$$

o bien el sistema

$$
\mathrm{CaO}-\mathrm{C}_{3} \mathrm{~S}-\mathrm{C}_{3} \mathrm{~A}-\mathrm{C}_{4} \mathrm{AF},
$$

con la siguiente composición potencial:

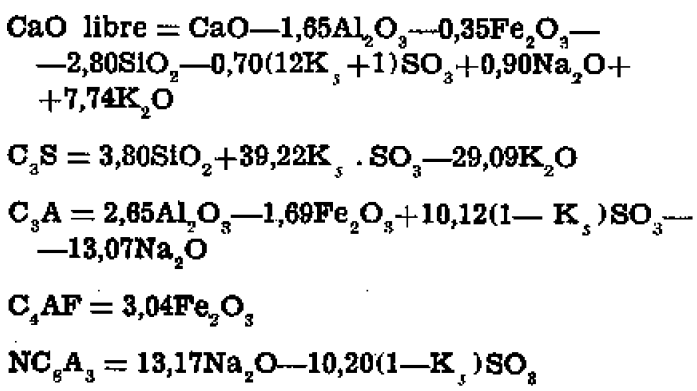

$\mathrm{KC}_{23} \mathrm{~S}_{\mathrm{z}^{2} \mathrm{z}}=22,35 \mathrm{~K}_{2} \mathrm{O}-26,30 \mathrm{~K}_{\mathrm{s}}, \mathrm{SO}_{3}$

$\mathrm{Na}_{2} \mathrm{SO}_{4}=1,77\left(1-\mathrm{K}_{s}\right) \mathrm{SO}_{8}$

$\mathrm{K}_{2} \mathrm{SO}_{4}=2,18 \mathrm{~K}_{3} . \mathrm{SO}_{3}$

Considerados dentro del cemento portland, los álcalis resultan ser muy nocivos para la integridad de los morteros y hormigones confeccionados con determinados tipos de áridos que se manifiestan reactivos frente a aquéllos, y que dan lugar a expansiones y desintegraciones de los materiales aglomerados. Además de esto, a la parte de álcalis soluble en agua (la que se encuentra en forma de sulfatos) es atribuíble la formación de eflorescencias $y$ los fallos de algunos recubrimientos hechos con morteros de cementos con contenido elevado de álcalis. En otro aspecto, hay ocasiones en que el falso fraguado del cemento va ligado a la presencia de álcalis en el mismo, los cuales se carbonatan en atmósfera húmeda; los carbonatos resultantes reaccionan después con el hidróxido cálcico liberado en la hidrólisis de los silicatos, precipitando carbonato cálcico.

El aspecto de la interacción áridos-alcalis, $y$ el de sus efectos sobre el hormigón, ha sido tratado con gran extensión, principalmente por los investigadores norteamericanos, con carácter más o menos general (10 a 37).

También se ha prestado gran atención a la interpretación del mecanismo físicoquímico que rige este tipo de interacciones (38 a 45), así como a los ensayos más idóneos para ponerlas de maniflesto $\mathrm{y}$ valorarlas ( 46 a 63), y a los métodos analíticos para establecer la cuantía de los álcalis y la proporción de ellos que se encuentran en una u otra forma (64 a 74). Finalmente, en cuanto a medidas preventivas, se ha abordado el problema de corregir la expansión debida a la acción entre los áridos reactivos y los álcalis del cemento (74 a 81).

\section{PARTE EXPERIMENTAL.}

Se ha estudiado la distribución de alcalis $\left(\mathrm{Na}_{2} \mathrm{O}+\mathrm{K}_{2} \mathrm{O}\right)$, por separado $\mathrm{y}$ en conjunto, en un clínker de cemento portland. Para ello se utilizó un material gra- 
nulado, obtenido por cocción con fuel-oll en un horno de tipo Lepol. Se eligió el clínker de este tipo de horno porque, dadas las características del mismo, el crudo de alimentación entra en 61 ya granulado a un tamaño bastante uniforme. En tales condiciones los gránulos mantienen su individualidad desde que entran en la parrilla hasta que salen clinkerizados, sin que prácticamente tenga lugar ninguna interacción mutua durante su paso por el horno.

En cuanto al combustible empleado para la clinkerización, el fuel-oil tiene Ia ventaja de no aportar cenizas que, incorporándose al crudo $o$, en el caso presente, formando un recubrimiento sobre los gránulos, pudieran falsear los resultados y la interpretación de los mismos.

El objeto perseguido fué determinar la variación del contenido de $6 x$ xido sódico y óxido potásico en zonas concéntricas de los gránulos, desde la periferla hasta el núcleo, es decir, a lo largo de un radio. Se pretendió con ello completar estudios ya realizados y ampliar resultados anteriormente obtenidos acerca de la distribución heterogénea de la cal libre en el granulado de clínker (82), y en relación con la estructura general de éste, en cuanto al reparto de componentes ácidos y básicos, así como de fases y constituyentes mineralógicos en las distintas zorras de los gránulos (83) (84) (85).

\subsection{Preparactón de las muestras.}

Para la separación de las distintas zonas de los gránulos se utilizó el procedimiento mecánico que se describe a continuación.

Se comenzó por seleccionar gránulos de un mismo tamaño, para lo cual se cribaron varios kilogramos de clínker granulado, empleando, sucesivamente, dos cribas de 1,25 cm y 1,50 cm de luz de malla (cuadrada). Se desechó la fracción que quedó sobre la primera y la que pasó a través de la segunda, quedando como válida la fracción comprendida entre ambos tamaños.
De ésta se eligieron, a continuación, los gránulos sensiblemente más esféricos, de los cuales, a su vez, se tomaron, al azar, 50.

De momento, cada uno de estos gránulos se consideró individualmente como un elipsoide y, mediante un calibre, se midieron los tres hipotéticos ejes del mismo.

Estos no resultaron en ningún caso muy distintos entre si, y por ello, y con objeto de asimilar los nódulos a esferas, se halló la media aritmética de los tres valores correspondientes a cada gránulo. Tampoco las medias así calculadas difirieron $\mathrm{mu}$ cho unas de otras, dada la selección previa, por lo cual se halló nuevamente la media de todas ellas, considerando el valor resultante como diámetro medio de los nódulos, supuestos éstos esféricos. El valor obtenido fué de $1,37 \mathrm{~cm}$, es decir, a los gránulos se les asignó un radio esférico de 0,685 centímetros.

Los 50 nódulos, cuyo peso global fué inicialmente de 201,1 gramos, se introdujeron en el vaso de hierro de un molino vibratorio, en ausencia de elementos molturadores, a fin de que el frotamiento de unos gránulos contra otros, resultante de la vibración, produjese su desgaste.

De tiempo en tiempo se abría el vaso y se extraia el polvo producido, el cual, en cada caso, constituyó la muestra correspondiente. En los casos en que resultaba partido algún gránulo, se eliminaban los trozos, los cuales no se volvian a introducir en el vaso en la siguiente operación. recogiendo sólo el polvo fino que pasaba por un tamiz de 4.900 mallas $/ \mathrm{cm}^{2}$ y considerándose el residuo sobre el tamiz, cuando lo había, con material procedente de fractura de gránulos y no de desgaste.

Con esta hipótesis el polvo extraído en cada operación, correspondiente, por lo tanto, a cada muestra, se supone procedente de todos los nódulos en la misma proporción, es decir, se admite que éstos se desgasten sensiblemente por igual, $\sin$ que ninguno sufra un desgaste desproporcionado con relación al de los demás. 
Se adoptó el criterio de considerar como periferia o corteza de los mismos la zona externa, con un peso de material equivalente a los 3/4 del peso total del gránulo, $o$, lo que es lo mismo, con un espesor equivalente a 1/3 del radio medio, y como núcleo la parte interna, con un peso equivalente al cuarto restante del peso del gránulo, es decir, con un espesor igual a los 2/3 restantes del radio.

Así, pues, los desgastes sucesivos de los gránulos se prolongaron hasta que el total del polvo extraído por nódulo fué aproximadamente de 3 gramos, dado que el peso medio de un gránulo fứe de 4 gramos (201,1:50), tentendo en cuenta los eliminados por rotura. Finalmente, se trituraron, en su totalidad, los gránulos restantes.

El cuadro 1 da idea de los resultados obtenidos con este procedimiento operato- rio. Las cifras de la columna 2 indican el número de nódulos que se tratan en cada operación sucesiva de desgaste; las de la 3 , el peso correspondiente a dichos nódulos; las de la 4, el número de nódulos que aparecen rotos al final de cada operación, y que no intervienen ya en la siguiente; las de la 5, el peso de dichos nódulos (incluído el del residuo que deja la muestra obtenida sobre un tamiz de $4.900 \mathrm{ma}-$ llas $/ \mathrm{cm}^{2}$ ); las de la 6 , el polvo extraído en cada operación (que pasa siempre, en su totalidad, por el tamiz de $4.900 \mathrm{ma}-$ llas $/ \mathrm{cm}^{2}$, salvo cuando hay rotura de gránulos), y que constituye cada una de las muestras; las de la 7, el polvo extraído de cada gránulo, en cada operación aislada, $y$, finalmente, las de la 8 ; el polvo total extraído de cada gránulo en las sucesivas operaciones, considerando acumulado el de todas las anteriores.

\section{CUA D R O 1}

\begin{tabular}{|c|c|c|c|c|c|c|c|}
\hline $\begin{array}{c}1 \\
\text { Muentra } \\
\text { nám. }\end{array}$ & $\begin{array}{c}2 \\
\text { Námiero } \\
\text { de n6dulos }\end{array}$ & $\begin{array}{c}\text { g } \\
\text { Pesóde } \\
\text { los nódulos } \\
\text { (gramos) }\end{array}$ & $\begin{array}{l}\text { Númeroro } \\
\text { de nódulos } \\
\text { roton }\end{array}$ & $\begin{array}{c}\text { Pesa de } \\
\text { Pos nódulos } \\
\text { notos } \\
\text { (gramos) }\end{array}$ & $\begin{array}{c}\text { 6 } \\
\text { Polvo } \\
\text { etraido } \\
\text { (gramos) }\end{array}$ & $\begin{array}{c}\text { Polvo } \\
\text { extrafido } \\
\text { por nodidalo } \\
\text { (gramos) }\end{array}$ & $\begin{array}{l}\text { Polva totad } \\
\text { extrafifo } \\
\text { par nfodulo } \\
\text { (Eramos) }\end{array}$ \\
\hline 1 & 50 & 201,10 & 0 & - & 7,0348 & 0,1407 & 0,1407 \\
\hline 2 & 50 & 194,06 & 0 & - & 7,2340 & 0,1447 & 0,2854 \\
\hline 3 & 50 & 186,83 & 0 & - & 6,6086 & 0,1322 & 0,4176 \\
\hline 4 & 50 & 180,22 & 0 & - & 4,5153 & 0,0903 & 0,5079 \\
\hline 5 & 50 & 175,71 & 1 & 3,53 & 4,7839 & 0,0957 & 0,6036 \\
\hline 6 & 49 & 167,40 & 0 & - & 4,5972 & 0,0938 & 0,6974 \\
\hline 7 & 49 & 162,80 & 0 & - & 4,5991 & 0,0939 & 0,7913 \\
\hline 8 & 49 & 158,20 & 0 & - & 5,2530 & 0,1072 & 0,8985 \\
\hline 9 & 49 & 152,94 & 1 & 3,14 & 4,8772 & 0,0995 & 0,9980 \\
\hline 10 & 48 & 144,93 & 3 & 9,15 & 4,7018 & 0,0980 & 1,0960 \\
\hline 11 & 45 & 131,10 & 0 & - & 4,5404 & 0,1009 & 1,1969 \\
\hline 12 & 45 & 126,53 & 1 & 2,85 & 4,9502 & 0,1100 & 1,3069 \\
\hline 13 & 44 & 118,73 & 0 & - & 6,0952 & 0,1385 & 1,4454 \\
\hline 14 & 44 & 112,64 & 2 & 5,18 & 11,3014 & 0,2569 & 1,7023 \\
\hline 15 & 42 & 96,20 & 0 & - & 9,4519 & 0,2250 & 1,9273 \\
\hline 16 & 42 & 86,71 & 4 & 8,44 & 4,8915 & 0,1165 & 2,0438 \\
\hline 17 & 38 & 73,40 & $\theta$ & 12,00 & 6,3470 & 0,1670 & 2,2108 \\
\hline 18 & 32 & 55,03 & 8 & 14,64 & 5,4030 & 0,1688 & 2,3796 \\
\hline 19 & 24 & 35,00 & 2 & 3,32 & 5,0986 & 0,2124 & 2,5920 \\
\hline 20 & 22 & 26,60 & 1 & 1,15 & 4,1221 & 0,1874 & 2,7794 \\
\hline 21 & 21 & 21,30 & 0 & - & 3,0305 & 0,1443 & 2,9237 \\
\hline 22 & 21 & 18,30 & 21 & - & 18,2633 & 0,8697 & 3,7934 \\
\hline
\end{tabular}


Como puede apreciarse, se prolongó el desgaste hasta obtener la muestra 21 de aproximadamente $3 \mathrm{~g}(2,9237 \mathrm{~g})$, equivalente a los $3 / 4$ del peso total de un nódulo, que es de unos $4 \mathrm{~g}$ (201,1:50; 4/3 . $\cdot 0,685^{3}$. 3; siendo: $201,1 \mathrm{~g}$ el peso total de los 50 gránulos, $0,685 \mathrm{~cm}$ el radio esférico medio de cada uno y 3 la densidad real aproximada del clínker).

Asi, pues, las distintas muestras 1 a 21 inclusive, corresponden a capas sucesivas de los gránulos, las cuales, por fijar ideas, se consideran como corticales, mientras que la 22 corresponde a la zona interna o núcleo de aquéllos.

\subsection{Cáleulo del espesor de las capas separadas.}

A partir de las cifras de polvo total extraído de cada gránulo (columna $8 \mathrm{del}$ cuadro 1) se calcularon los espesores de las zonas separadas por desgaste en cada operación, de la forma siguiente: si es $r_{n}(n=1,2 \ldots 21)$ el radio medio de los gránulos al cabo de la $n$-sima operación, la disminución media del volumen de los gránulos al final de la misma será:

$$
\frac{4}{3} \pi\left(0,685^{3}-r_{n}^{3}\right)
$$

siendo 0,685 el radto medto inicial de los gránulos. Este volumen corresponde al del polvo total $\mathbf{P}_{n}$ extraído al cabo de la n-sima operación de desgaste (cifras de la columna 8 del cuadro 1), y considerando igual a 3 el peso específtco real del clínker, se tiene

$$
\frac{4}{3} \pi\left(0,685^{3}-r_{n}^{3}\right)=\frac{P_{n}}{3},
$$

de donde se deduce el valor de $r_{n}$ :

$$
r_{n}=\sqrt[3]{\frac{4,037-\vec{P}_{n}}{12,56}}
$$

\begin{tabular}{|c|c|c|c|}
\hline$\stackrel{1}{1}$ Muestrám. & 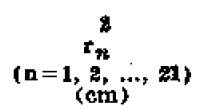 & $\begin{array}{c}\mathbf{E}_{n}{ }^{3} \\
(\mathrm{n}=1, \ldots, \ldots, 21\} \\
(\mathrm{em})\end{array}$ & 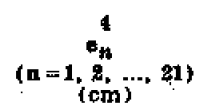 \\
\hline & $r_{\mathrm{v}}=0,685$ & $E_{0}=0$ & $e_{0}=0$ \\
\hline 1 & $r_{1}=0,676$ & $\mathrm{E}_{3}=0,009$ & $e_{1}=0,009$ \\
\hline 2 & $r_{2}=0,668$ & $\mathrm{E}_{2}=0,017$ & $e_{2}=0,008$ \\
\hline 3 & $r_{3}^{2}=0,661$ & $\mathrm{E}_{\mathrm{s}}=0,024$ & $e_{3}=0,007$ \\
\hline 4 & $\tau_{4}=0,655$ & $E_{4}=0,030$ & $e_{4}=0,006$ \\
\hline 5 & $r_{5}=0,649$ & $\mathbf{E}_{3}=0,036$ & $e_{3}=0,006$ \\
\hline 6 & $r_{0}=0,644$ & $E_{i \mathrm{i}}=0,041$ & $e_{6}=0,005$ \\
\hline 7 & $r_{7}^{8}=0,637$ & $E_{7}=0,048$ & $e_{8}^{b}=0,007$ \\
\hline 8 & $r_{s}=0,630$ & $\mathbf{E}_{s}=0,055$ & $e_{8}=0,007$ \\
\hline 9 & $r_{\mathrm{g}}=0,624$ & $\mathrm{E}_{\mathrm{g}}^{\mathrm{g}}=0,06 \mathrm{I}$ & $e_{\mathrm{a}}=0,006$ \\
\hline 10 & $r_{10}=0,616$ & $\mathbf{E}_{10}=0,069$ & $e_{20}=0,008$ \\
\hline 11 & $r_{11}=0,610$ & $\mathbf{E}_{\mathbf{1 1}}=0,074$ & $e_{11}=0,005$ \\
\hline 12 & $r_{72}=0,601$ & $\mathbf{E}_{12}=0,084$ & $e_{w^{2}}=0,010$ \\
\hline 13 & $r_{x z}=0,591$ & $\mathrm{E}_{13}=0,094$ & $e_{18}=0,010$ \\
\hline 14 & $r_{14}=0,571$ & $\mathrm{E}_{\mathrm{i}, 4}=0,114$ & $e_{14}=0,020$ \\
\hline 15 & $r_{I 5}=0,552$ & $E_{15}=0,133$ & $e_{1 s}=0,019$ \\
\hline 16 & $r_{16}=0,542$ & $\mathrm{E}_{16}=0,143$ & $e_{10}^{u}=0,010$ \\
\hline 17 & $r_{17}=0,526$ & $E_{17}=0,159$ & $e_{17}=0,016$ \\
\hline 18 & $r_{18}^{17}=0,509$ & $E_{18}=0,176$ & $e_{13}^{18}=0,017$ \\
\hline 19 & $r_{10}=0,486$ & $\mathrm{E}_{1 \mathrm{~g}}=0,199$ & $e_{18}=0,023$ \\
\hline 20 & $r_{20}=0,464$ & $\mathbf{E}_{20}=0,221$ & $e_{20}=0,022$ \\
\hline 21 & $r_{21}=0,446$ & $\mathrm{E}_{21}=0,239$ & $e_{9 q_{1}}=0,018$ \\
\hline 22 & - & - & \\
\hline
\end{tabular}

CUADEO 2 
Sustituyendo $\mathrm{P}_{n}$ por los distintos valores del cuadro 1 se obtienen los radios medios $r_{n}$ resultantes para los gránulos al cabo de cada operación, los cuales se indican en la columna 2 del cuadro 2 . Por diferencia entre estos radios $r_{n}$ y $r_{o}=0,685$ se obtienen los espesores $\mathbf{E}_{n}$ totales de las zonas separadas por desgaste hasta la n-sima operación (columna 3 del cuadro 2). Estos espesores son, por lo tanto, "acumulados", como corresponde a los valores de $P_{n}$, que son también cantidades de polvo extraídas "acumuladas". Por diferencia entre cada dos valores sucesivos de $E_{n}$ se obtienen los espesores medios $e_{n}$ correspondientes a las zonas separadas por desgaste en cada operación aislada (Columna 4 del cuadro 2).

Como puede apreciarse, el espesor medio total desgastado en los gránulos fué de $0,239 \mathrm{~cm}$, lo que supone aproximadamente 1/3 del radio medio inicial de los gránulos $(0,685: 3=0,228)$, parte que, convencionalmente, se considera como corteza; el resto, $0,446 \mathrm{~cm}$, supone los dos tercios restantes $(0,456)$, y la parte correspondiente (muestra 22) se considera como núcleo.

\subsection{Analisis de las muestras.}

En cada una de las 22 muestras obtenidas se determinó el contenido de álcalis, óxidos sódico y potásico, por separado.

Se empleó para ello un fotómetro de llama tipo Lange, modelo 5 , con filtros para la determinación selectiva de sodio o de potasio. Se siguió en líneas generales el método operatorio descrito por la correspondiente norma norteamericana (86) si bien se utilizó como combustible gas del alumbrado.

El calibrado previo del aparato para el trazado de la curva intensidad-concentración se hizo mediante disoluciones patrón de concentraciones de sodio y potasio conocidas, con un contenido de calcio muy aproximado al del problema, dado que en éste forzosamente se han de en- contrar los cationes alcalinos en presencia de otros, entre los cuales el calcio es el más abundante. Se da, ađemás, la circunstancia de que la linea espectral Ca I $586 \mathrm{~m}_{\mu}$ es muy próxima a la $\mathrm{Na} 589 \mathrm{~m}_{\mu}$, por lo que se produce siempre una interferencia que, en este caso, se soslaya recurriendo al arbitrio señalado.

En cuanto a la influencia del silicio - componente del clínker que sigue en orden de abundancia al calcio-, ensayos llevados a cabo eliminando la sílice en el problema, y también sin eliminarla, parecen demostrar que tan sólo tiene una influencia de cierta magnitud en los resultados relativos al sodio, cuando el contenido de éste en el clínker es muy pequeño, en cuyo caso procede eliminar la sílice (87).

El aluminio, el hierro y el magnesio que contiene el clínker, en las proporciones usuales en que se encuentran en éste, no parecen interferir las radiaciones del sodio, potasio y calcio; si bien el aluminio, en proporeiones mayores, deprime la radiación del calcio. En todo caso, puede eliminarse el efecto de esta acción depresora operando como en el caso del calcio, es decir, haciendo que la disolución patrón contenga aluminio en análoga proporción a la que contiene normalmente el clínker.

Las disoluciones de reserva de sodio $y$ potasio, así como la de calcio, y las disojuciones patrón obtenidas a partir de ellas, se prepararon siguiendo las prescripciones del método preconizado por las normas A. S. T. M. (86).

El calibrado del fotómetro se hizo observando las instrucciones propias del aparato, y se trazaron las correspondientes curvas en las que cada división del eje de abscisas representaba un $0,01 \%$ de metal alcalino en forma de óxido; en ordenadas se representaron divisiones de la escala del galvanómetro.

El ataque y disolución por ácido clorhídrico de cada una de las muestras 1 a 22 (columna 6 del cuadro 1), así como la determinación del óxido sódico y potásico 
en ellas, utilizando en cada caso el filtro correspondiente a cada catión, se efectuó igualmente según la norma norteamericana (86) (88).

Con objeto de obtener datos para efectuar los cálculos que se detallan en el apartado 4 , se hizo el análisis completo de algunas de las muestras (las 2, 4, 5, 7, $12,13,14,19$ y 22) siguiendo las prescripciones de la correspondiente norma $\mathbf{A}$. S. T. M. para cemento. Los análisis se llevaron a cabo sobre las muestras calcinadas.

(Continuará.)

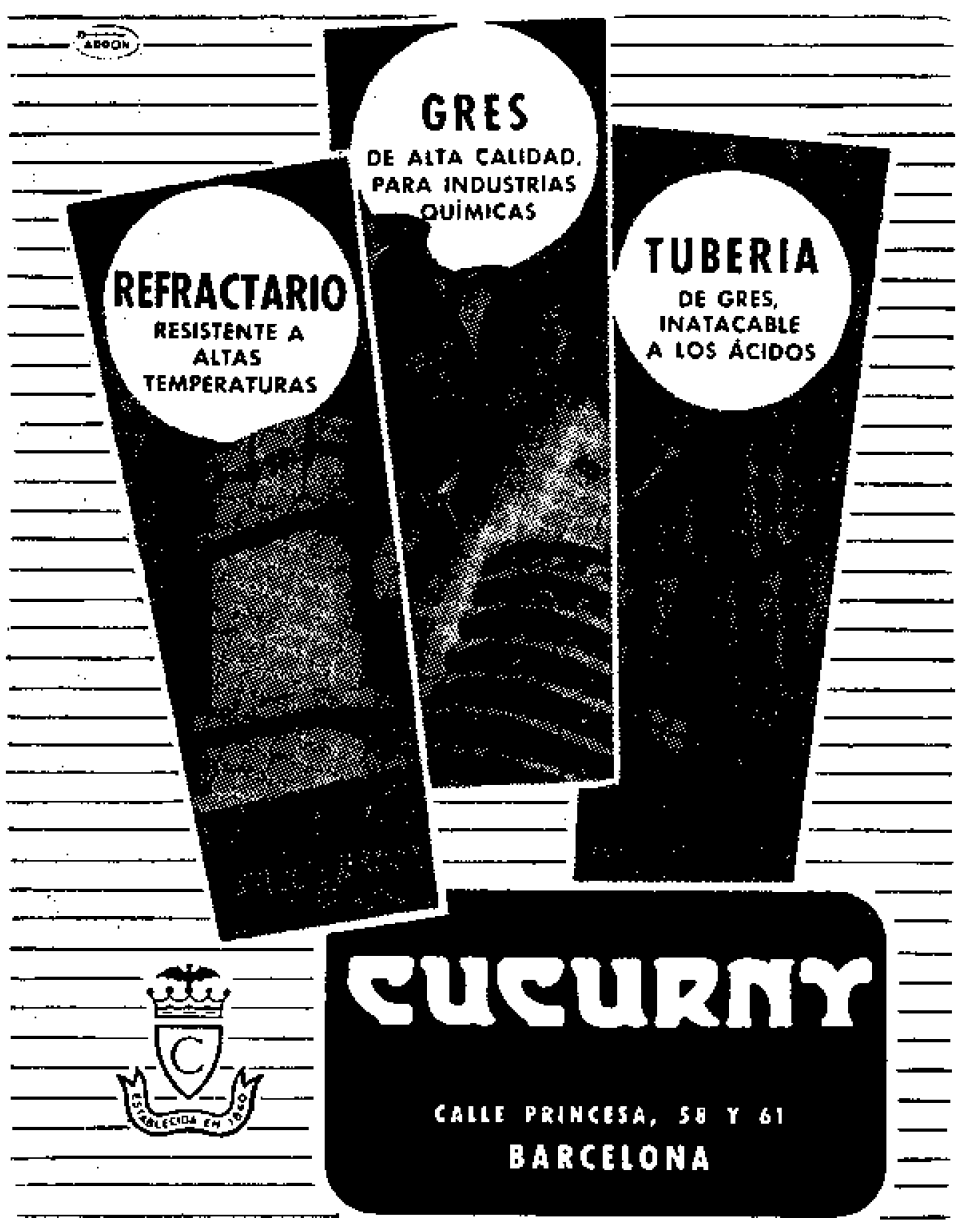

